\title{
On Valuing Appreciating Human Assets in Services
}

\author{
O. Zeynep Akşin \\ Koç University \\ Rumeli Feneri Yolu, 34450 Sariyer-Istanbul, Turkey \\ +90 212338 1545, fax: +90 212338 1653, zaksin@ku.edu.tr \\ January 2003; revised November 2004; revised May 2006
}




\title{
On Valuing Appreciating Human Assets in Services
}

\begin{abstract}
This paper derives optimal policies for when to hire, train and layoff employees in service organization when worker productivity appreciates deterministically or stochastically under constant, monotonic, and cyclic service demand. The model, an extension of the machine replacement problem from the engineering economy literature, uses infinite horizon linear programming to produce optimal personnel planning strategies and dual prices that characterize the workforce's economic value. The effect of employee attrition is also considered. In its valuation of human assets, unlike pure accounting approaches that only try to measure, this paper proposes an approach to capture the interaction between firm decisions pertaining to human resources and human resource value.

Keywords: Service operations, human resource valuation, depreciation, manpower planning, learning, training decisions, mathematical programming.
\end{abstract}




\section{Introduction}

Recent years have seen downsizing or large-scale job cutting become a permanent aspect of corporate strategy $([12,31])$. Three types of downsizing strategies are identified by [37]: workforce reduction strategies that eliminate individual jobs, organizational redesign related downsizing that eliminates entire departments or layers, and systemic employment strategy that views downsizing as a continuous opportunity. Except for organizational redesign strategies, downsizing requires decisions regarding whom to lay off, when and in what quantities to lay off. Should a company keep its experienced, older workforce or should it focus its energies on young employees? Does economic rationality call for laying off large numbers of the inexperienced employees who haven't been invested in much, or is it better to lay off experienced employees who require higher compensation? Some criteria used by companies are last-in-first-out, the removal of everybody below a certain level in the hierarchy, or the weeding out of all middle managers [13]. According to survey results in [37], there is not a single formula or practice adopted by managers. However the importance of objective criteria which can be defended in cases of illegal discrimination are emphasized [43].

Turnover, the voluntary quitting of a firm by its employees, has significant costs for companies [20], especially in knowledge intense settings [34]. Human resource managers need to understand the impact of turnover on the value of their employees and be able to compare the costs and benefits of different turnover reduction policies. Both the downsizing and the turnover problem point to the need for information that captures the economic consequences of decisions pertaining to human resources. It is this issue that motivates the ensuing analysis. A modeling framework is developed to assess the value of human assets for service companies. In the case of downsizing, this approach will provide an objective means of evaluating the value of an individual to a firm over the long-term.

For service firms it is not always the case that labor is a variable cost [17]. Motivated by David Ricardo, [41] analyzes the value of services in three different components: labor, human capital and physical capital. Under this classification, human capital is information or knowledge and experience whereas labor is the unskilled portion of manpower. It is observed that information, training or knowledge do not perish under normal circumstances, hence a depreciation in the physical sense does not occur. Human capital is said to depreciate, once the learning curve of an employee flattens with respect to others. Note that this depreciation is purely in the market sense. 
Today, a growing portion of the service sector workforce is classified as knowledge workers. The increasing knowledge content of work has instigated a stream of research that looks at the valuation of intangible assets $([9,53,44])$. The new mix of employees suggests that more emphasis needs to be placed on the knowledge or information content of human resources, abandoning the traditional view of labor as variable costs. Under the new paradigm, human resources are treated as an asset of the firm. This implies that one time hiring and training costs need to be spread out over the economic life of employees at a firm, preferably in a way that reflects their economic value. This argument parallels the traditional theory for the depreciation of physical capital. A significant difference between physical and human capital is that people are capable of learning and improving their performance over time. Hence, the use of the term "appreciation" schedules in this paper.

Our aim herein, is to characterize the economic value of human resources to the firm. Establishing the relationship between human resource value and personnel policies will enable the analysis of downsizing and turnover reduction problems in a long-term time frame. Thus for example, a manager who suddenly finds out that a large number of employees from a particular job need to be removed, will be able to use the valuation in guiding his decision on who will be laid off. The ultimate decision will be driven by many different things not considered herein, however the valuation will help the manager in focusing on some employees through an objective and economically sound criterion.

The following section provides a review of some related literature, and positions the approach in this paper with respect to existing work in accounting and operations management. The basic model is developed in Section 3. This model is analyzed in Section 4. Section 5 establishes the results on employee appreciation. Section 6 considers the assumption of deterministic learning made in the basic model, and illustrates the implications of relaxing this assumption. Numerical examples are presented in Section 7. The paper ends with concluding remarks.

\section{Literature Review}

The basic question that has been addressed by human resource accounting (HRA) research is whether humans are assets or merely an expense to the firm. Starting out from this question, [47], [52] classify HRA research into two basic approaches. The historical cost approach, or ex post valuation, values humans through the recruiting, selection, training 
and separation costs incurred by the firm. Time related issues pertaining to an individual's tenure in the firm and mobility of human resources that establish the system dynamics have to be taken into account. The economic theory approach, also referred to as ex ante valuation, tries to estimate the firm's future earnings, account for discounting and allocate present value to human resources. In determining the payoff schedule to the firm, one has to decide if human resources depreciate or appreciate over time.

In a critique of HRA, [49] point out that the valuation models developed thus far substitute measurable but invalid surrogate measures for human resource value. A variety of HRA models have been developed and occasionally implemented at companies $([7,25,35,16])$. A depreciation scheme for human resources is proposed in [26] and [33]. A survey of the HRA literature by [48] provides a broad classification of existing studies.

Different approaches for intangible resource valuation are reviewed in [6]. As pointed out in their paper, the main weakness of HRA models is that everything concerning cost, human resource mobility, and value needs to be estimated and as such the resulting valuation is quite subjective. The approach that is proposed in this paper addresses this problem of HRA models. The human resource mobility is modeled through a manpower flow model, and the valuation is based on optimal decisions from this model. This ensures an objective valuation, given basic data about costs, training, productivity, and attrition. A depreciation schedule for human resources that can be derived from this new valuation scheme is described. To this end we formulate a manpower planning model that makes some standard assumptions regarding human resource dynamics in a firm $([55,24,1])$, and additionally incorporates the effects of learning.

The impact of learning on productivity improvement has been formalized in the learning curve literature. A comprehensive survey of the literature can be found in [8] and [57]. Manpower scheduling is affected by the learning curve phenomenon. Work has been done to plan for manpower requirements in the presence of learning by $[56,51,29]$. A manpower planning problem is formulated as a partial differential equation model in [21], that accounts for learning through an increase in productivity. In order to incorporate the effects of learning in our model, we take an approach similar to theirs and readers are referred to their paper for a more detailed analysis of the learning curve.

Learning has also been incorporated in aggregate planning models. The productivity of workers is assumed to increase with increased tenure in the model of [42]. The effects of learning on productivity in aggregate production planning models are also considered 
by $[11,30]$. Large scale manpower planning models using mathematical programming have been extensively used in military applications [36, 10], as reviewed in [19]. A separate stream of research, focusing on Markov Chain models, incorporates learning in manpower planning, however the focus is on forecasting personnel inventory levels rather than controlling personnel decisions [2]. Some exceptions are noted in a recent paper [18], which considers the optimal hiring decision under learning and turnover formulated as a Markov Deciosion Problem.

The model in this paper has been motivated by the machine replacement and capacity expansion problem developed by [27]. The latter model is extended in several ways herein. Rather than assuming a fixed depreciation rate, the firm in this paper is able to control the appreciation of employee productivity through training decisions. Workers can be hired at different experience levels, thus generalizing the new machine purchasing decision found in [27]. Employee attrition is modeled, corresponding to a machine failure in the capital equipment replacement setting. This was not modeled in [27]. Finally, this paper considers optimal hiring and training decisions in the presence of constant, monotonic, and cyclic demand. The consideration of different demand types is another extension of [27].

In summary, the model in this paper combines ideas from HRA and manpower planning models. As in HRA, human resources are considered as assets of the firm and the focus is on human resource valuation. However this valuation is based on optimal personnel policies determined by a manpower planning model, as opposed to observed or projected policies. While pure accounting approaches only try to measure, the approach proposed in this paper captures the interaction between firm decisions and actions pertaining to human resources and human resource value. This paper is primarily concerned with the enginnering economy question of how long to keep employees as they learn and gain skill. The consideration of this issue within a mathematical program for workforce planning is unique.

\section{The Model}

In this section, an infinite horizon manpower planning model is formulated that addresses hiring, training and layoff of personnel for a certain job in the service sector. Similar models for infinite horizon problems can be found in [24]. The model treats every employee as an identical, individual entity. Thus all employees at a particular experience level are assumed to have identical productivity. Behavioral factors and personal differences are 
ignored, mostly because the aim is to come up with aggregate level personnel policies. This implies that the model being considered is appropriate for organizations that are large enough (for example 100 or more employees) where such averaging will make sense. Using the classification in [54] the model considers observable sources of heterogeneity, which are length of service and training as explained below, and ignores latent sources of heterogeneity. We look at personnel policies in the presence of constant, monotonic increasing, monotonic decreasing, and cyclic service demand. Stationary policies are shown to exist for the first three types of demand. These results are then extended to obtain some general policy guidelines for the cyclic case.

A single type of job is considered. This job can be one found in a call center or in a shared services organization, consisting of a standard set of tasks. Employees can be at different levels of experience, depending on the time they have spent on the job. As in [46], aging and experience are related. A similar assumption is made in [18], motivated by a call center example. A zero year old employee is someone who has no working experience on the job, like a new graduate for example. Every year, the employee gains one year's worth of experience. Stated otherwise, learning occurs through experience and through on-the-job-training, which is extensively studied in [4]. The gain in experience is reflected in an increased productivity rate. Many models in the labor economics literature make a similar assumption (see for example [32, 40]). While years and time periods are used interchangeably throughout the paper, these periods can be shorter than a year. For a job where employees tend to stay for a long time, years would be appropriate, whereas for a job where employees stay only for several years (in a call center for example), months or quarters would be the appropriate interval.

The firm is able to invest in an employee at any time by training her. It is assumed that training changes where the employee is operating on the learning curve, thus resulting in a change in productivity. For ease of exposition, it has been assumed that training a person will expedite her by one year's worth of experience. Thus, a person who is trained with zero years of experience will start work at a productivity level of one year old employees. It is assumed that she will be paid the wages of a one year old employee. This assumption implies a perfectly mobile workforce and will be relaxed in future research. It is clear that one can equally well consider the case when training results in productivity increases that cannot be achieved through experience alone. The associated cost of training depends on the current years of experience that the employee has. The firm incurs all the costs from training and no deductions are made in the trainee's wages. Note that since the time periods 
are years, training has been assumed to have instantaneous effects. If one year's experience worth of training requires a significant amount of time, this may imply that productivity in years with training would be expected to be lower. However since the model assumes all empoyees are identical within a cohort, it is not possible to distinguish the productivity of individuals who have received training and those who have not in the same group. A different formulation of the problem may be required to deal with this effect. Thus, only the case where training constitutes a short duration relative to the length of the time periods being considered is modeled herein. The basic assumption of deterministic learning will be relaxed in Section 6.

An employee can be hired at any level of experience. It is assumed that hired personnel start work at the beginning of the period in which they were hired. The hiring cost can change for hiring personnel with different years of experience. This enables the firm to supply some of its service requirements from the outside, as opposed to training existing personnel, or waiting for them to gain experience.

Every year existing employees can either be kept or laid off. The third option, which is training the employee was mentioned before. Although we use the term layoff throughout the paper, these movements can be interpreted differently. Since they constitute part of a pre-planned replacement cycle for an employee, they are in essence a jump in the career path, which may at times represent an internal transfer within the firm, or early retirement. These layoffs should not be confused with sudden large-scale downsizing initiatives that are typically beyond the control of manpower planners. The cost of keeping an employee is the wages associated with a particular level of experience. Laying off results in a cost which is once again dependent on the level of experience of the person being laid off. It includes the costs due to the inefficiencies that occur when replacements take place, and related processing costs. For a detailed analysis of these costs, see [45]. We assume for simplicity that the firm has no severance plans for laid off employees, hence attrition costs are identical to layoff costs. Layoffs occur at the end of a period.

The rate at which employees are lost due to voluntary quits is called the attrition rate. As in [55], these attrition rates have been incorporated into the personnel flow constraints (1) and (2) of the model. These rates are typically based on historical data available in the firm or similar firms. We assume an upper bound on the maximum number of years any employee will work on the job that is being considered herein. This can be interpreted as a time after which the employee does not find it desirable to work in that position, and 
automatically leaves.

Every year a certain amount of service requirements have to be met by the personnel. These requirments are derived from company forecasts and supplied as parameters to the model.

We introduce the following notation:

$n \equiv$ limiting age (years of experience) beyond which an employee does not stay;

$w_{j} \equiv$ wages paid to an employee with $j$ years of experience, $j=0, \ldots, n-1$;

$h_{j} \equiv$ hiring cost of hiring an employee with $j$ years of experience, $j=0, \ldots, n-1$;

$r_{j} \equiv$ layoff cost for an employee with $j$ years of experience, $j=1, \ldots, n$;

$I_{j} \equiv$ investment made to train a $j$ year old employee to $j+1$ years of experience, $j=0, \ldots, n-2$;

$K_{j} \equiv$ productivity rate of an employee with $j$ years of experience, $j=0, \ldots, n-1$;

$S_{t} \equiv$ units of total service required in year $t$, for $t=1,2, \ldots$;

$\alpha \equiv$ discount factor, $0<\alpha<1$;

$\mathbf{b}_{\mathbf{0}} \equiv$ a vector of size $n$ denoting the initial distribution of the number of employees available at the beginning of period 1 with $j$ years of experience, $j=0, \ldots, n-1$;

$\mathbf{C}=\left[w_{1}-r_{1}, \ldots, w_{n-1}-r_{n-1}\left|h_{0}+w_{0}, \ldots, h_{n-1}+w_{n-1}\right|\right.$ $\left.I_{1}+w_{2}-r_{1}, \ldots, I_{n-2}+w_{n-1}-r_{n-2} \mid I_{0}+w_{1}+h_{0}, \ldots, I_{n-2}+w_{n-1}+h_{n-2}\right]$

A row vector of size $4 n-4$ for the cost of respectively keeping (modified by a contribution to layoff costs by those who are kept), hiring, keeping and immediately training (modified by a contribution to layoff costs by those who are trained) hiring and immediately training, an employee with different years of experience.(Includes layoff cost contributions implicit in the flow constraints (1) and (2), as demonstrated below);

$\mathbf{D}=\left[r_{2}, \ldots, r_{n}\left|r_{1}, \ldots, r_{n}\right| r_{3}, \ldots, r_{n} \mid r_{2}, \ldots, r_{n}\right]$

A row vector of size $4 n-4$ that accounts for the remaining part of the layoff costs. It will be shown that $\mathbf{C}$ does not account for the total layoff cost;

$\mathbf{K}=\left[K_{1}, \ldots, K_{n-1}\left|K_{0}, \ldots, K_{n-1}\right| K_{2}, \ldots, K_{n-1} \mid K_{1}, \ldots, K_{n-1}\right]$

A row vector of size $4 n-4$ for the productivity rates of respectively keeping, 
hiring, keeping and immediately training, hiring and immediately training an employee with corresponding years of experience;

$$
\mathbf{A}=\left[\begin{array}{c|c|c|c}
I^{n-1} & 0 & I^{n-2} & 0 \\
0 & 0 & 0 & 0
\end{array}\right]
$$

An $n$ by $4 n-4$ matrix, where $I^{n-1}$ denotes the identity matrix of order $n-1$;

$$
\mathbf{B}=\left[\begin{array}{c|c|c|c}
0 & I^{n} & 0 & 0 \\
I^{n-1} & & I^{n-2} & I^{n-1}
\end{array}\right]
$$

An $n$ by $4 n-4$ matrix, where $I^{n}$ denotes the identity matrix of order $n$. The $\mathbf{B}$ matrix changes when we consider attrition rates as well. In this case all values in row $i$ are multiplied by $p_{i}$ (the survival rate), where $1-p_{i}$ is the attrition rate for $i=1, \ldots, n$. Similar modifications are performed on the initial distribution vector $b_{0}$.

$\mathbf{X}_{\mathbf{t}} \equiv$ a column vector of size $4 n-4$ with $X_{t}(j)$ for $j=1, \ldots, n-1$ denoting the employees that are kept with $j$ years of experience, for $j=n, \ldots, 2 n-1$ the employees that are hired with $j-n$ years of experience, for $j=2 n, \ldots, 3 n-3$ the employees that are trained with $j-2 n+1$ years of experience and for $j=3 n-2, \ldots, 4 n-4$ the employees that are hired and immediately trained with $j-3 n+2$ years of experience $(t=1,2, \ldots)$

The model becomes a minimization of the sum of discounted costs over an infinite horizon, subject to service requirement and personnel flow constraints. The summation over an infinite horizon avoids end effects that may distort the dual price value over a finite horizon. It assumes that the firm has an infinite lifetime, which is consistent with the literature on the theory of the firm in economics. We obtain the following model:

$$
\begin{array}{crr}
\min & \sum_{t=1}^{\infty} \alpha^{t-1}\left[\mathbf{C X}_{\mathbf{t}}+\alpha \mathbf{D} \mathbf{X}_{\mathbf{t}}\right]+\mathbf{r} \mathbf{b}_{\mathbf{0}} & \\
\text { s.t. } & \mathbf{K X}_{\mathbf{t}} \geq S_{t} & \\
& \mathbf{A X}_{\mathbf{1}} \leq \mathbf{b}_{\mathbf{0}} & \\
\mathbf{A X}_{\mathbf{t}} \leq \mathbf{B X}_{\mathbf{t}-\mathbf{1}} & t=1,2, \ldots \\
\mathbf{X}_{\mathbf{t}} \geq 0 & t=1,2, \ldots
\end{array}
$$


Notice that the decision variable $\mathbf{X}_{\mathbf{t}}$ does not have any component for the number of employees that are laid off in any year. These numbers are obtained implicitly, using the personnel flow constraints (1) and (2). To demonstrate this point, a set of the flow constraints will be written out for a certain period $t$. For simplicity, a superscript is added to the variable $\mathbf{X}_{\mathbf{t}}$ denoting the different components: $k$ for employees that are kept, $h$ for employees that are hired, $d$ for employees that are trained, $h d$ for employees that are hired and trained, and $l$ for those that are laid off. Assume that all attrition rates are equal to zero. For $n=4$ the following constraints are obtained:

$$
\begin{aligned}
X_{t-1}^{h}(0) & \geq X_{t}^{k}(1)+X_{t}^{d}(1) \\
X_{t-1}^{k}(1)+X_{t-1}^{h}(1)+X_{t-1}^{h d}(0) & \geq X_{t}^{k}(2)+X_{t}^{d}(2) \\
X_{t-1}^{k}(2)+X_{t-1}^{h}(2)+X_{t-1}^{d}(1)+X_{t-1}^{h d}(1) & \geq X_{t}^{k}(3) \\
X_{t-1}^{k}(3)+X_{t-1}^{h}(3)+X_{t-1}^{d}(2)+X_{t-1}^{h d}(2) & \geq 0 .
\end{aligned}
$$

The number of people laid off is then determined as

$$
\begin{aligned}
& X_{t}^{l}(1)=X_{t-1}^{h}(0)-X_{t}^{k}(1)-X_{t}^{d}(1) \\
& X_{t}^{l}(2)=X_{t-1}^{k}(1)+X_{t-1}^{h}(1)+X_{t-1}^{h d}(0)-X_{t}^{k}(2)-X_{t}^{d}(2) \\
& X_{t}^{l}(3)=X_{t-1}^{k}(2)+X_{t-1}^{h}(2)+X_{t-1}^{d}(1)+X_{t-1}^{h d}(1)-X_{t}^{k}(3) \\
& X_{t}^{l}(4)=X_{t-1}^{k}(3)+X_{t-1}^{h}(3)+X_{t-1}^{d}(2)+X_{t-1}^{h d}(2)
\end{aligned}
$$

The layoff costs have been incorporated into the objective function, using these equalities as a conversion for the variables. For each variable $X_{t}^{l}(i)$ there is an associated cost of $r_{i}$ $(i=1, \ldots, n)$. Thus the contribution of layoff costs to the objective function becomes

$$
\begin{aligned}
& r_{1} X_{t}^{l}(1)=r_{1} X_{t-1}^{h}(0)-r_{1} X_{t}^{k}(1)-r_{1} X_{t}^{d}(1) \\
& r_{2} X_{t}^{l}(2)=r_{2} X_{t-1}^{k}(1)+r_{2} X_{t-1}^{h}(1)+r_{2} X_{t-1}^{h d}(0)-r_{2} X_{t}^{k}(2)-r_{2} X_{t}^{d}(2) \\
& r_{3} X_{t}^{l}(3)=r_{3} X_{t-1}^{k}(2)+r_{3} X_{t-1}^{h}(2)+r_{3} X_{t-1}^{d}(1)+r_{3} X_{t-1}^{h d}(1)-r_{3} X_{t}^{k}(3) \\
& r_{4} X_{t}^{l}(4)=r_{4} X_{t-1}^{k}(3)+r_{4} X_{t-1}^{h}(3)+r_{4} X_{t-1}^{d}(2)+r_{4} X_{t-1}^{h d}(2)
\end{aligned}
$$

The layoff costs associated with year $t$ have been subtracted from the corresponding components of $\mathbf{C}$ and the ones for year $t-1$ have been collected in the cost vector $\mathbf{D}$. Since $\mathbf{r b}_{\mathbf{0}}$ is a constant, we will drop it from the objective function in the subsequent analysis. 


\section{Solving the Infinite Horizon Problem}

The infinite horizon linear programming problem has been considered before. Due to the lack of a general duality theory, it remains as a difficult problem to solve ([22, 23, 14, 24]). We adopt a similar solution technique to that in [27].

\subsection{Constant, Monotonically Increasing or Decreasing Demand}

Given the primal infinite-horizon problem in the preceding section, we obtain the dual as follows:

$$
\begin{aligned}
\max \mu_{1} \mathbf{b}_{\mathbf{0}}+\sum_{t=1}^{\infty} \lambda_{t} S_{t} & \\
\text { s.t. } \lambda_{t} \mathbf{K}+\mu_{t} \mathbf{A}-\mu_{t+1} \mathbf{B} & \leq \alpha^{t-1}(\mathbf{C}+\alpha \mathbf{D}) \quad \forall t=1,2, \ldots \\
\lambda_{t} & \geq 0 \\
\mu_{t} & \leq 0
\end{aligned}
$$

Let $\left\{\mathbf{X}_{\mathbf{t}}\right\}_{t=1}^{\infty}$ be a solution of the primal infinite horizon problem, and assume additionally that the right hand sides of the primal constraints are $\alpha$-convergent. Specifically let

$$
\lim _{T \rightarrow \infty} \sum_{t=1}^{T} \alpha^{t-1} S_{t}=S<\infty .
$$

This assumption states that the discounted sum of service requirements converges to a finite value. For constant demand it holds trivially, given $\alpha<1$. Define

$$
\beta=\left\{\bar{b} \in R^{n} \mid \bar{b}(i) \geq 0 \text { for } i=0, \ldots, n-1, \sum_{i=0}^{n-1} K_{i} \bar{b}(i)=S\right\}
$$

such that $\mathbf{B X}_{\mathbf{t}-\mathbf{1}} \in \beta$ (this is true since one will never keep-hire more workforce than needed) and $\mathbf{b}_{\mathbf{0}} \in \beta$. Setting $X=\sum_{t=1}^{\infty} \alpha^{t-1} \mathbf{X}_{\mathbf{t}}$, multiplying the $t^{t h}$ constraint set by $\alpha^{t-1}$, and summing over $t$, we introduce the following one period finite primal/dual LP pair $P_{1}(\bar{b}, \bar{\mu}, S)$ and $D_{1}(\bar{b}, \bar{\mu}, S)([23,27])$ :

$$
\left(P_{1}\right) \quad \min (C X+\alpha D X+\alpha \bar{\mu} B X)
$$




$$
\begin{aligned}
\text { s.t. } K X & \geq S \\
A X & \leq \bar{b} \\
X & \geq 0 \\
\text { s.t. } \lambda K+\mu A & \leq C+\alpha D+\alpha \bar{\mu} B \\
\lambda & \geq 0 \\
\mu & \leq 0
\end{aligned}
$$

Theorem 1 If there exists a $\bar{\mu}$ and $\bar{\lambda}$ (stationary dual prices) that solve $D_{1}(\bar{b}, \bar{\mu}, S)$ for all $S$, then the sequence $\left\{X_{t}\right\}_{t=1}^{\infty}$ defined by the rule

$$
\begin{array}{lll}
\mathbf{X}_{\mathbf{1}} & \text { solves } & P_{1}\left(b_{0}, \bar{\mu}, S_{1}\right) \\
\mathbf{X}_{\mathbf{t}} & \text { solves } & P_{1}\left(B X_{t-1}, \bar{\mu}, S_{t}\right) \text { for } \quad t=2,3, \ldots
\end{array}
$$

solves the infinite horizon primal problem, and $\left\{\alpha^{t-1} \bar{\lambda}\right\}_{t=1}^{\infty},\left\{\alpha^{t-1} \bar{\mu}\right\}_{t=1}^{\infty}$ solve the dual problem.

(All proofs can be found in the Appendix). To apply this theorem to our problem, we must characterize a $\bar{\mu}$ that solves the dual problem $D_{1}$. Let $m_{i}^{k}(j), j=i, \ldots, k-1$ be a vector of zeroes and ones of size $(k-i-1)$, where a one denotes a training and a zero a no training decision in a period. Denote by $M$ the set of feasible training policies $m_{i}^{k} \in M$. Let $\bar{m}_{i}^{k}(j)=\sum_{l=i}^{j} m_{i}^{k}(l)$ and $j^{\prime}=j+\bar{m}_{i}^{k}(j-1)$. Define the following:

$$
\begin{gathered}
E_{i}^{k}=\sum_{j=i}^{k-1} \alpha^{j-i}\left(m_{i}^{k}\left(j^{\prime}\right)\left(I_{j^{\prime}}+w_{j^{\prime}+1}\right)+\left(1-m_{i}^{k}\left(j^{\prime}\right)\right)\left(w_{j^{\prime}}\right)\right), \\
P_{i}^{k}=\sum_{j=i}^{k-1} \alpha^{j-i}\left(m_{i}^{k}\left(j^{\prime}\right)\left(K_{j^{\prime}+1}\right)+\left(1-m_{i}^{k}\left(j^{\prime}\right)\right)\left(K_{j^{\prime}}\right)\right) .
\end{gathered}
$$

In calculating these summations, we take $w_{s}=0$ for $s>n$. Then

$$
\begin{aligned}
& \left(j, l, n_{j}^{l}\right)= \\
& \arg \min _{\left(i, k, m_{i}^{k}\right)}\left\{\frac{h_{i}+E_{i}^{k}+\alpha^{k-i-\bar{m}_{i}^{k}(k-1)} r_{k}}{P_{i}^{k}} \mid i=0, \ldots, n-1, k=1, \ldots, n, i<k ; m_{i}^{k} \in M\right\}
\end{aligned}
$$


The expression (3) represents the present value (in terms of cost of one unit of service) of hiring an $i$ year old person, keeping him, training him according to policy $m_{i}^{k}$, and laying him off when he becomes $k$ years old. Thus, $j, l$, and $n_{j}^{l}$ are the values of $i, k$ and $m_{i}^{k}$ that minimize this cost.

Now let

$$
\tilde{\lambda}=\frac{h_{j}+E_{j}^{l}+\alpha^{l-j-\bar{n}_{i}^{l}(l-1)} r_{l}}{P_{j}^{k}}
$$

where we set $m_{j}^{l}=n_{j}^{l}$. This is the minimum cost of one unit of service, hence we can state that the dual variable $\bar{\lambda}$ is equal to $\tilde{\lambda}$. Here $j$ stands for the age an employee starts working, and $l$ is the age she is laid off, based on the policy selected in determining $\tilde{\lambda}$. We can now define $\bar{\mu}$ as

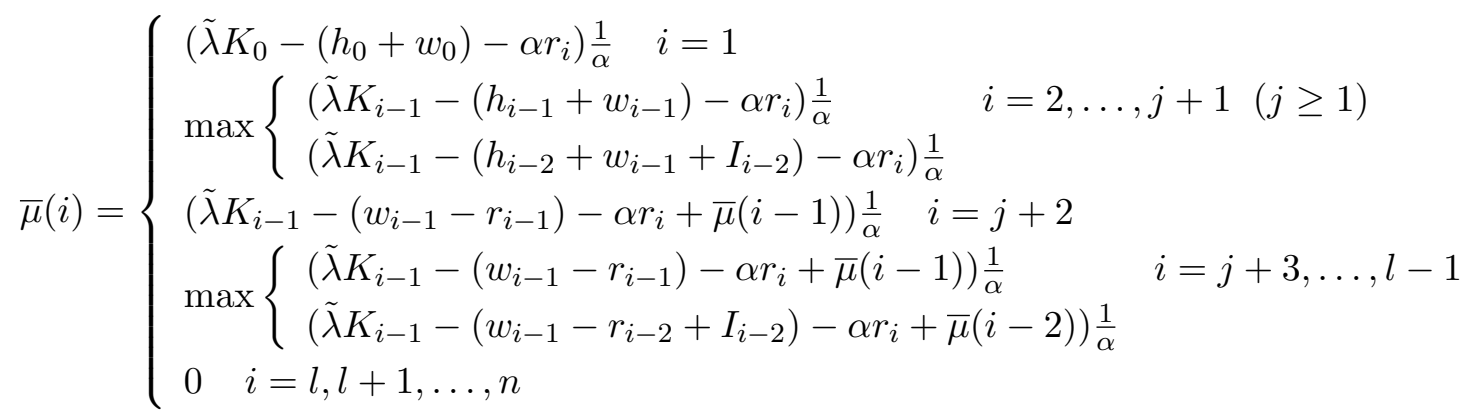

Theorem $2 \bar{\mu}$ as characterized above solves the dual problem $D_{1}(\bar{b}, \bar{\mu}, S)$ for all $S$ and $\bar{b} \in \beta$.

Let us now consider the case where $1-p_{i}$ is the attrition rate associated with $i$ year old employees $(i=1, \ldots, n)$. As noted before, the matrix $\mathbf{B}$ will change, such that row $i$ is multiplied by $p_{i}$. The dual variables $\bar{\lambda}$ and $\bar{\mu}$ are re-characterized to account for attrition rates. First observe that for $i, k$, and $m_{i}^{k}$ as defined above, the least cost policies are obtained by

$$
\begin{gathered}
\left(j, l, n_{j}^{l}\right)=\arg \min _{\left(i, k, m_{i}^{k}\right)} \\
\left\{\frac{h_{i}+E_{i}^{k}+R_{i}^{k}+\alpha^{k-i-\bar{m}_{i}^{k}(k-1)}\left(\prod_{s=i}^{k-2} p_{s}\right) r_{k}}{P_{i}^{k}} \mid i=0, \ldots, n-1, k=1, \ldots, n, i<k ; m_{i}^{k} \in M\right\}
\end{gathered}
$$

where

$$
E_{i}^{k}=\sum_{j=i}^{k-1} \alpha^{j-i}\left(m_{i}^{k}\left(j^{\prime}\right)\left(\prod_{s=i}^{j^{\prime}} p_{s}\right)\left(I_{j^{\prime}}+w_{j^{\prime}+1}\right)+\left(1-m_{i}^{k}\left(j^{\prime}\right)\right)\left(\prod_{s=i}^{j^{\prime}-1} p_{s}\right)\left(w_{j^{\prime}}\right)\right)
$$




$$
\begin{gathered}
R_{i}^{k}=\sum_{j=i}^{k-1}\left(\alpha^{j-i+1}\left(1-p_{j}\right)\left(\prod_{s=i}^{j-1} p_{s}\right) r_{j}\right), \\
P_{i}^{k}=\sum_{j=i}^{k-1} \alpha^{j-i}\left(m_{i}^{k}\left(j^{\prime}\right)\left(\prod_{s=i}^{j^{\prime}} p_{s}\right)\left(K_{j^{\prime}+1}\right)+\left(1-m_{i}^{k}\left(j^{\prime}\right)\right)\left(\prod_{s=i}^{j^{\prime}-1} p_{s}\right)\left(K_{j^{\prime}}\right)\right) .
\end{gathered}
$$

In these calculations, we take $p_{s}=1$ whenever $m_{i}^{k}(s)=1$. Due to the attrition rates $\left(1-p_{i}\right)$, we incur attrition costs every year, but pay less for wages and training (by a factor of $p_{i}$ ) since the chance of attrition accumulates over the employee's tenure. Using the above definitions, $\bar{\lambda}$ is determined as

$$
\bar{\lambda}=\frac{h_{j}+E_{j}^{l}+R_{j}^{l}+\alpha^{l-j-\bar{n}_{j}^{l}(l-1)}\left(\prod_{s=j}^{l-2} p_{s}\right) r_{l}}{P_{j}^{l}}
$$

The age an employee starts working $(j)$ and is laid off $(l)$ and the training poicy $n_{i}^{k}$ is determined as before. The new characterization of $\bar{\mu}$ will be of the form

$$
\bar{\mu}(i)=\left\{\begin{aligned}
&\left(\tilde{\lambda} K_{0}-\left(h_{0}+w_{0}\right)-\alpha r_{i}\right) \frac{1}{\alpha p_{1}} \quad i=1, \quad j=0 \\
&\left(\tilde{\lambda} K_{0}-\left(h_{0}+w_{0}\right)-\alpha r_{i}\right) \frac{1}{\alpha} \quad i=j=1 \\
& \max \begin{cases}\left(\tilde{\lambda} K_{i-1}-\left(h_{i-1}+w_{i-1}\right)-\alpha r_{i}\right) \frac{1}{\alpha} & i=2, \ldots, j \quad(j \geq 2)\end{cases} \\
& \max \begin{cases}\left(\tilde{\lambda} K_{i-1}-\left(h_{i-1}+w_{i-1}\right)-\alpha r_{i}\right) \frac{1}{\alpha p_{i}} & i=j+1 \quad j \geq 1 \\
\left(\tilde{\lambda} K_{i-1}-\left(h_{i-2}+w_{i-1}+I_{i-2}\right)-\alpha r_{i}\right) \frac{1}{\alpha p_{i}} & i=j+3, \ldots, l-1\end{cases} \\
&\left(\tilde{\lambda} K_{i-1}-\left(w_{i-1}-r_{i-1}\right)-\alpha r_{i}+\bar{\mu}(i-1)\right) \frac{1}{\alpha p_{i}} \quad i=j+2 \\
& \max \left\{\begin{array}{l}
\left(\tilde{\lambda} K_{i-1}-\left(w_{i-1}-r_{i-1}\right)-\alpha r_{i}+\bar{\mu}(i-1)\right) \frac{1}{\alpha p_{i}} \\
\left(\tilde{\lambda} K_{i-1}-\left(w_{i-1}-r_{i-2}+I_{i-2}\right)-\alpha r_{i}+\bar{\mu}(i-2)\right) \frac{1}{\alpha p_{i}} \\
0 \quad i=l, l+1, \ldots, n
\end{array}\right.
\end{aligned}\right.
$$

Theorem 1 can now be applied to solve for the optimal primal sequence $\left\{X_{t}\right\}_{t=1}^{\infty}$. The resulting dual prices will be of the form $\left\{\alpha^{t-1} \bar{\lambda}\right\}_{t=1}^{\infty}$ and $\left\{\alpha^{t-1} \bar{\mu}\right\}_{t=1}^{\infty}$, thus stationary except for the discount factor $\alpha^{t-1}$. 


\subsection{The Case of Cyclic Demand}

Let us now consider the case where the demand for services is not constant or monotonic, but follows the pattern of business cycles. In particular, assume that demand varies periodically, repeating a cycle every $q$ periods. For any integer $i$ and $j$ with $|i-j|=t q, \quad t=1,2, \ldots$, we will have $S_{i}=S_{j}$. Note that one can also consider the case where there is an increasing (or decreasing) trend in the periodic demand. Then for $i<j$, with $i$ and $j$ defined before, we get $S_{i}<S_{j}$ or $\left(S_{i}>S_{j}\right)$. Setting

$$
\bar{X}_{j}=\alpha^{j-1} X_{j}+\alpha^{j+q-1} X_{j+q}+\alpha^{j+2 q-1} X_{j+2 q}+\ldots \quad j=1,2, \ldots, q
$$

assuming

$$
\lim _{T \rightarrow \infty} \sum_{t=0}^{T} \alpha^{j+t q-1} S_{j+t q}=\bar{S}_{j}<\infty \text { for } j=1,2, \ldots, q
$$

and defining

$$
\beta_{j}=\left\{\bar{b}_{j} \in R^{n} \mid \bar{b}_{j}(i) \geq 0 \text { for } i=0, \ldots, n-1, \sum_{i=0}^{n-1} K_{i} \bar{b}_{j}(i)=\bar{S}_{j}\right\}, j=1, \ldots, q
$$

we obtain the $q$ period primal/dual problems, $P_{q}\left(\bar{b}_{j}, \bar{\mu}_{j}, S_{j}\right)$ and $D_{q}\left(\bar{b}_{j}, \bar{\mu}_{j}, S_{j}\right)$. The above notation implies that $\bar{X}_{j}$ is the discounted sum of the recurring policies $(j=1, \ldots, q)$, over an infinite horizon. When compared to the constant or monotonic demand case, we observe that the series converges to a repeating cycle of $q$ different policies, instead of a single stationary policy. The assumption regarding the structure of the demand data, undergoes a similar change when compared to the constant/monotonic demand case. For constant cyclic demand, the convergence result is trivial. If there is an increasing trend to the cyclic demand, it may not always be the case that the summations converge to a finite value. Thus, for such cases the assumption is a restrictive one. For ease of notation, let the lagging periods $q+1$ and 0 denote periods 1 and $q$ respectively throughout this section.

$$
\begin{array}{cr}
\left(P_{q}\right) \quad \min \sum_{j=1}^{q}\left(\mathbf{C} \bar{X}_{j}+\left(\mathbf{D}+\bar{\mu}_{j+1} \mathbf{B}\right) \alpha \bar{X}_{j-1}\right) \\
\text { s.t. } \quad \mathbf{K} \bar{X}_{j} \geq \bar{S}_{j} \quad j=1, \ldots, q \\
\quad \overline{\mathbf{X}}_{j} \leq \bar{b}_{j} \quad j=1, \ldots, q \\
\bar{X}_{j} \geq 0 \quad j=1, \ldots, q
\end{array}
$$




$$
\begin{array}{rll}
\left(D_{q}\right) & \max \sum_{j=1}^{q} \lambda_{j} \bar{S}_{j}+\mu_{j} \bar{b}_{j} & \\
\text { s.t. } \quad & \lambda_{j} \mathbf{K}+\mu_{j} \mathbf{A} \leq \mathbf{C}+\alpha \mathbf{D}+\alpha \bar{\mu}_{j+1} \mathbf{B} & j=1, \ldots, q-1 \\
\lambda_{j} \mathbf{K}+\mu_{j} \mathbf{A} & \leq \mathbf{C}+\alpha \mathbf{D}+\alpha \bar{\mu}_{1} \mathbf{B} & j=q \\
\lambda_{j} & \geq 0 & j=1, \ldots, q \\
\mu_{j} & \leq 0 & j=1, \ldots, q
\end{array}
$$

Periodic problems of infinite horizon have been dealt with before. The dynamic programming literature (see, for example, [5]) provides results showing that the solutions to these types of problems are of a periodic stationary nature. More specifically [3] show that for an infinite horizon capacity expansion problem, periodic demand gives rise to cyclic optimal policies. Applying these results to the human resource problem we conclude that the optimal policy will be a periodic repetition of replacement cycles. In particular, let $S=\left\{S_{1}, S_{2}, \ldots, S_{q}\right\}, \bar{b}=\left\{\bar{b}_{1}, \bar{b}_{2}, \ldots, \bar{b}_{q}\right\}, \bar{\mu}=\left\{\bar{\mu}_{1}, \ldots, \bar{\mu}_{q}\right\}$, and $\bar{\lambda}=\left\{\bar{\lambda}_{1}, \ldots, \bar{\lambda}_{q}\right\}$. The notation $\tilde{t}$ is used to denote the remainder of the integer division of $t$ by $q$.

Theorem 3 If there exists a $\bar{\mu}$ and $\bar{\lambda}$ that solve $D_{q}(\bar{b}, \bar{\mu}, S)$ for all $S$, then the sequence $\left\{X_{t}\right\}_{t=1}^{\infty}$ defined by the rule

$$
\begin{array}{lllll}
X_{1} & \text { solves } & P_{q}\left(\bar{b}_{0}, \bar{\mu}_{2}, S_{1}\right) \\
X_{k q+j} & \text { solves } & P_{q}\left(\mathbf{B} X_{j-1}, \bar{\mu}_{j+1}, S_{j}\right), \quad \text { for } \quad k=0, j=2, \ldots, q \\
X_{k q+j} & \text { solves } & P_{q}\left(\mathbf{B} X_{j-1}, \bar{\mu}_{j+1}, S_{j}\right), \quad \text { for } \quad k=1,2, \ldots, j=1, \ldots, q
\end{array}
$$

solves the infinite horizon primal problem, and $\left\{\alpha^{t-1} \bar{\lambda}_{\tilde{t}}\right\}_{t=0}^{\infty},\left\{\alpha^{t-1} \bar{\mu}_{\tilde{t}}\right\}_{t=0}^{\infty}$ solve the dual problem.

This result implies that given a periodic demand structure, the optimal policy eventually becomes cyclic. Instead of a single stationary policy, the firm will now have periodically fluctuating hiring, training and firing policies.

Recall that for the cases of constant and monotonic demand, we identified the best replacement cycle, based on cost minimization. The cost of one unit of service was then determined, using this optimal replacement cycle. Since it is assumed that none of the cost or productivity parameters of the model change when demand becomes periodic, we observe that the optimal replacement cycle remains the same. However, due to the cyclic nature of 
the demand, we will have decreasing demand at some points in the cycle. This implies a necessity to lay off large quantities of employees. The model will lay off according to the optimal replacement cycle, but may have to lay off some employees before the optimal age. The value of the dual variables will change, depending on the resulting layoff policies. Thus, for the case of cyclic demand, we are not able to characterize the dual variables analytically. Numerical analysis is required to determine these values.

\section{Analysis of Employee Appreciation}

This section analyzes the way in which employees rise or fall in value over their tenure in the firm. We define appreciation (depreciation) schedules based on the economic interpretation of the stationary dual prices.

The parameter $\tilde{\lambda}$ is the cost of one unit of service for a repeated cycle of identical hirings, trainings (it may also be the case that engaging in no training is preferable) and replacements. The term $\alpha \bar{\mu}(i)$ is the value of having an $i-1$ year old employee in the previous period. The problem is a cost minimization and as a result the value of an employee can be interpreted as negative costs. Thus if the decision is to keep an $i-1$ year old employee, $\alpha \bar{\mu}(i)$ is defined as the value of an $i-1$ year old $\alpha \tilde{\lambda} K_{i-1}$, minus the costs involved in keeping that person $\left(w_{i-1}-r_{i-1}+\alpha r_{i}\right)$, plus the value of having an $i-2$ year old employee the previous period. The decisions of hiring and training can be explained in a similar fashion, based on the definition of $\bar{\mu}$.

The depreciation schedule is defined so that book value is equal to the stationary dual price in each time period. Hence we obtain

$$
\begin{aligned}
d_{1}=\bar{\mu}(1) & j & =0 \\
d_{i}=\bar{\mu}(i-1)-\bar{\mu}(i), & i & =j, j+1, \ldots, l
\end{aligned}
$$

where $d_{i}$ is the depreciation to be charged in year $i$. Note that for negative, increasing $\bar{\mu}, d_{i}$ also becomes negative. This indicates that employees are appreciating (negative depreciation) over time. Using the definition of $\bar{\mu}$, and denoting the costs (outlays) of period $i$ by $O_{i}, d_{i}$ is equivalently characterized as

$$
-\frac{1}{\alpha} \tilde{\lambda} K_{i-1}+\frac{1}{\alpha} O_{i-1}+\left(\frac{\alpha-1}{\alpha^{i-1}}\right) \bar{\mu}(i-1)
$$

For the cyclic case, yearly depreciation figures can be calculated in a similar fashion, once the values of the $\bar{\mu}(i)$ 's are determined. 
Depreciation is a function of the costs and the productivity factors $K_{i}$. It is difficult to draw general results from these expressions analytically, due to the number of parameters involved. It should however be noted that they enable a sensitivity analysis based on varying costs or productivity factors. As an example, if the firm tries a new method of training which involves lower costs possibly by having employees pay part of it, one can measure its consequences on depreciation.

\section{When Learning is Not Deterministic}

In this paper, learning is modeled through an increase in productivity. So far, this increase is assumed to occur deterministically. This section explores the issue of when this assumption is valid and proposes a modification in the model for cases when this assumption would be too restrictive. The objective is not to provide a detailed analysis of stochastic learning, but to provide enough detail in order to illustrate the impact of this assumption on the paper's basic results. Stochastic learning has been modeled before in different settings. We refer the reader to [39] for a production planning problem with stochastic learning, modeled as an infinite horizon dynamic programming problem, and to [18] for hiring decisions in the presence of stochastic learning, modeled as a Markov Decision Problem. As demonstrated by these papers, a dynamic programming approach seems more appropriate to deal with the stochastic learning case.

In order to address the question of when a deterministic learning assumption may be appropriate, we draw on [38], and use a firm taxonomy discussed in that book. This taxonomy is used to relate firm strategy to the task environment in which a firm operates. Accordingly, firms can be classified in two groups, prospectors and defenders. The first group has firms that operate in a volatile environment, are innovative, and compete on being on the cutting edge rather than on efficiency or cost minimization. The second group has firms that defend existing markets and market share, are in more mature and stable settings, and tend to be more reactive than proactive in their strategies. Using this classification scheme, one can argue that the human resource valuation problem, as modeled so far, reflects the case of a defender firm. Given the relatively mature and stable setting, the problems faced by the firm will be well known and good solutions to these problems will have been developed. Appropriate selection and training practices will exist, ensuring a good fit between the deterministic learning assumption and reality. For such a firm, 
the current objective function that minimizes costs, and the assumption of deterministic improvements in productivity with learning will be appropriate. As one moves closer to the prospector end of the spectrum, the problems will be less well defined and understood, and as a result the relationship between experience and learning as well as training programs and learning will be less certain. In this case, it will be harder for the firm to specify selection and training practices that work. A stochastic learning assumption will be more appropriate. The firm objective will be to maximize the value of its employees.

More specifically, one can redefine $K_{i}$ for $i=0, \ldots, n-1$ as the revenue that can be generated by an employee with $i$ years of experience. The objective of the firm will be to maximize discounted total revenues less costs for an employee, over an infinite horizon. An individual's mobility in the firm is again determined by personnel flow constraints. The latter are modified to reflect the probabilistic nature of learning. Instead of assuming an increase in productivity with probability one, one can assume a Markovian structure wherein a probability transition matrix determines the probability of moving to a superior level of productivity subsequent to one year on the job (a keep decision) or a training investment (an investment decision). The tranistion probabilities are denoted by

$$
\begin{aligned}
\pi^{k}(i, j)= & \text { the probability that an employee that is kept in state (year) } i \text { will be in state } \\
& j \text { the following year }(i, j=0, \ldots, n), \text { where } \pi^{k}(i, j)=0 \text { if } j<i \text {, and } \\
& \pi^{k}(i, i)<1 \text { for all } i<n, \\
\pi^{d}(i, j)= & \text { the probability that an employee that is trained in state (year) } i \text { will be in } \\
& \text { state } j \text { the following year, where } \pi^{d}(i, j)=0 \text { if } j<i \text {, and } \\
& \pi^{d}(i, i)<1 \text { for all } i<n .
\end{aligned}
$$

The problem takes a form that is structurally similar to the machine replacement problem in [28], with an additional set of decisions concerning the training of an employee. The optimal replacement policy will again be stationary. The notation in Section 4 is changed as follows. For an employee hired in year $\tau \in\{0, \ldots, n-1\}$ let $\sigma_{\tau}^{k}$ be a vector of zeros and ones, with $\sigma_{\tau}^{k}(i)=1$ representing a keep decision and $\sigma_{\tau}^{k}(i)=0$ representing a layoff decision. Similarly, let $\sigma_{\tau}^{d}$ be a vector of zeros and ones, with $\sigma_{\tau}^{d}(i)=1$ representing a train decision and $\sigma_{\tau}^{d}(i)=0$ representing a do not train decision $\left(\sigma_{\tau}^{d}(n-1)=0\right)$. The expected present cost of having an employee with $i$ years of experience $i=\tau, \ldots, n-1$, training him according to training policy $\sigma_{\tau}^{d}(i)$ and replacing him according to replacement policy $\sigma_{\tau}^{k}(i)$ 
can be written as

$$
\begin{aligned}
E P C_{i}\left(\sigma_{\tau}^{k}, \sigma_{\tau}^{d}\right)= & \left(\sigma_{\tau}^{k}(i)\right)\left(\sigma_{\tau}^{d}(i)\right)\left(w_{i}+I_{i}+\alpha \sum_{j=i}^{n} \pi^{d}(i, j) E P C_{j}\left(\sigma_{\tau}^{k}, \sigma_{\tau}^{d}\right)\right)+ \\
& \left(\sigma_{\tau}^{k}(i)\right)\left(1-\sigma_{\tau}^{d}(i)\right)\left(w_{i}+\alpha \sum_{j=i}^{n} \pi^{k}(i, j) E P C_{j}\left(\sigma_{\tau}^{k}, \sigma_{\tau}^{d}\right)\right)+\left(1-\sigma_{\tau}^{k}(i)\right)\left(-r_{i}\right),
\end{aligned}
$$

with $\operatorname{EPC}_{n}\left(\sigma_{\tau}^{k}, \sigma_{\tau}^{d}\right)=-r_{n}$. Similarly, let the expected revenue generated by an employee currently in state $i$, employed over a replacement and training cycle $\left(\sigma_{\tau}^{k}, \sigma_{\tau}^{d}\right)$ be

$$
\begin{aligned}
\delta_{i}\left(\sigma_{\tau}^{k}, \sigma_{\tau}^{d}\right)= & \left(\sigma_{\tau}^{k}(i)\right)\left(\sigma_{\tau}^{d}(i)\right)\left(K_{i}+\alpha \sum_{j=i}^{n-1} \pi^{k}(i, j) \delta_{j}\left(\sigma_{\tau}^{d}, \sigma_{\tau}^{d}\right)\right) \\
& +\left(\sigma_{\tau}^{k}(i)\right)\left(1-\sigma_{\tau}^{d}(i)\right)\left(K_{i}+\alpha \sum_{j=i}^{n-1} \pi^{k}(i, j) \delta_{j}\left(\sigma_{\tau}^{k}, \sigma_{\tau}^{d}\right)\right) \\
& i \in\{\tau, \ldots, n-2\}
\end{aligned}
$$

where $\delta_{n-1}\left(\sigma_{\tau}^{k}, \sigma_{\tau}^{d}\right)=\sigma_{\tau}^{k}(n-1)\left(K_{n-1}+\alpha K_{n-1} \sigma_{\tau}^{k}(n-1) \pi^{k}(n-1, n-1)\right)$. Then

$$
\tilde{\lambda}_{\tau}=\min _{\sigma_{\tau}^{k}, \sigma_{\tau}^{d}} \frac{h_{\tau}+E P C_{\tau}\left(\sigma_{\tau}^{k}, \sigma_{\tau}^{d}\right)}{\delta_{\tau}\left(\sigma_{\tau}^{k}, \sigma_{\tau}^{d}\right)}
$$

and

$$
\tilde{\lambda}=\tilde{\lambda}_{\tau^{\star}}
$$

where

$$
\tau^{\star}=\operatorname{argmin}_{0 \leq \tau \leq n-1} \tilde{\lambda}_{\tau}
$$

In this case $\tilde{\lambda}$ represents the cost of generating one unit of revenue. Note the similarity between this expression and the one in Equation (5) under deterministic learning with attrition. In the stochastic learning setting, the transition probabilities have a similar role to the survival rates in Section 4. The numerical examples in the following Section explore the impact of attrition on human resource value. We expect stochastic learning, as modeled herein, to have a qualitatively similar impact. The analysis of this impact is not pursued any further here, given the parallels to the one in Section 4. For a treatment of depreciation in the stochastic learning case, readers are referred to [28].

\section{Numerical Examples}

A numerical example, first for the case of constant demand and then for cyclic demand is presented. The aim of this section is to illustrate the use of the approach and to explore the impact of cyclic demand on human resource value and appreciation. 


\begin{tabular}{|l|l|l|l|l|l|l|l|l|l|}
\hline \hline \multicolumn{2}{|c|}{$w$} & \multicolumn{2}{c|}{$h$} & \multicolumn{2}{c|}{$r$} & \multicolumn{2}{c|}{$I$} & \multicolumn{2}{c|}{$K$} \\
\hline yr. & wage & yr. & hire cost & yr. & layoff cost & yr. & training cost & yr. & prod. \\
\hline 0 & 2000 & 0 & 1000 & 1 & 1900 & 0 & 1000 & 0 & 0.5 \\
1 & 3000 & 1 & 2900 & 2 & 2600 & 1 & 2500 & 1 & 3 \\
2 & 7000 & 2 & 5000 & 3 & 4700 & 2 & 2500 & 2 & 4 \\
3 & 9000 & 3 & 6000 & 4 & 5800 & 3 & 2500 & 3 & 4.5 \\
4 & 10000 & 4 & 8000 & 5 & 6000 & 4 & - & 4 & 5 \\
\hline \hline
\end{tabular}

Table 1: Cost and Productivity Data for the Example

\begin{tabular}{|l|lllll|}
\hline \hline Scenario & $1-p_{1}$ & $1-p_{2}$ & $1-p_{3}$ & $1-p_{4}$ & $1-p_{5}$ \\
\hline 0 & 0 & 0 & 0 & 0 & 0 \\
\hline 1 & 0.40 & 0.25 & 0.15 & 0.10 & 0.05 \\
\hline 2 & 0.05 & 0.10 & 0.15 & 0.25 & 0.40 \\
\hline \hline
\end{tabular}

Table 2: Attrition Rates for the Three Scenarios

Consider the following problem where $n=5, \alpha=0.9$. Cost and productivity data are as given in Table 1. This example will be called the base case. Three sets of attrition rates as tabulated in Table 2 are considered. In Scenario 0, there is no attrition. In Scenario 1 less experienced employees leave with higher probabilities. Scenario 2 is the reverse case where they tend to leave in higher proportions as they become more tenured.

Service demand is assumed to be a constant 1000 units per period. Using the procedure in Section 4 the optimal replacement cycle is found to be the same for all three attrition scenarios. It is optimal for the firm to hire employees with zero years of experience and train them immediately. All employees leave when they reach the experience level of five years, which is the age beyond which an employee does not work. The value of one unit of service, $\bar{\lambda}$, is 2147, 2189, and 2179 for Scenarios 0, 1 and 2 respectively. An increase in the value of one unit of service in Scenarios 1 and 2 reflects the effect of attrition costs. The value of one unit of service is slightly less in Scenario 2 compared to Scenario 1 because for this particular example (costs and productivity parameters), it is more advantageous to lose employees as they become more experienced.

The dual variables and resulting depreciation schedule are shown in Figure 1. The 
graphs plot the negative of these values, which indicate appreciation. This appreciation may be attributed to the fact that the yearly productivities of employees are increasing over time, with respect to their costs. Notice that initially the employees appreciate, then in year three they depreciate and start appreciating again until they leave. The depreciation in year three indicates that costs of keeping the employee exceed their gain in productivity. However this depreciation does not induce a replacement of these employees. The potential appreciation in years four and five makes it economically desirable to keep the employees for two more years. For this example, a look at the depreciation schedule might instigate a revision of the compensation policy. We consider different wage alternatives below.
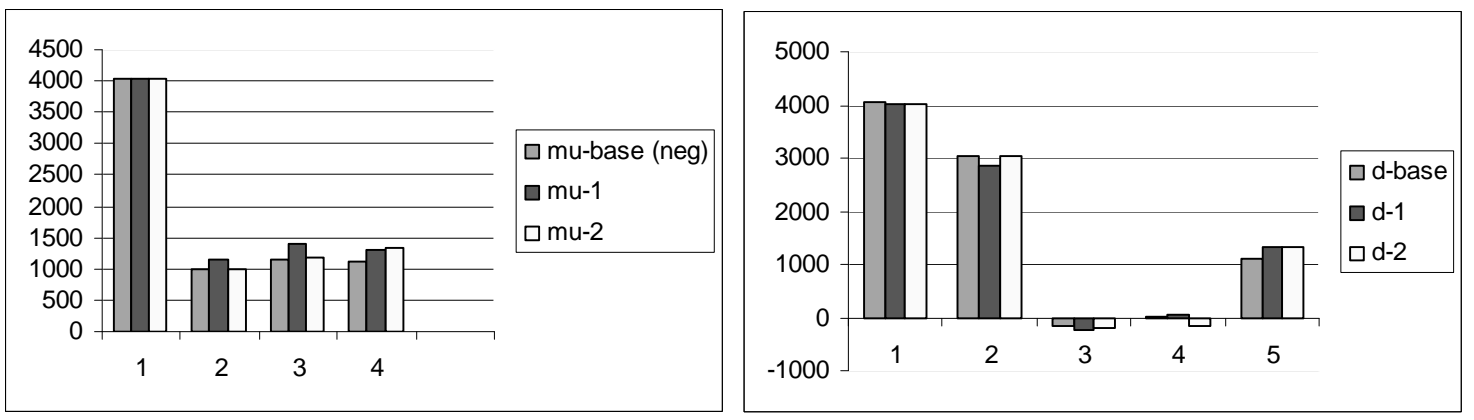

Figure 1: The dual variables $\mu$ and depreciation schedule for the example

Comparing Scenarios 0 and 1, we observe smaller appreciation and higher depreciation initially. This is due to the high rates of attrition for young employees. In years four and five, an employee appreciates more compared to the case without attrition. This reflects the difficulty in retaining employees as they become more experienced. For Scenario 2 we again have less appreciation in the first two years, when compared to the case without attrition. Initial appreciation is higher for this scenario since younger employees are easier to retain this time, but decreases as attrition increase with experience.

Consider the wages shown in Table 3 as potential alternatives to the one in the base case. These have been constructed such that wage ${ }^{1}$ avoids the excessive growth found in the base case wages, wage $e^{2}$ is proportional to the productivity vector, and wage $^{3}$ combines both characteristics. To explore the sensitivity of the optimal policy to the parameters, in addition to the base training costs $\left(I^{0}\right)$ we further consider two additional training cost vectors that are constructed such that training costs are 0.2 times the wage $\left(I^{0.2}\right)$ or 0.8 times the wage $\left(I^{0.8}\right)$. All new examples are considered under the three attrition rate scenarios found in Table 2. Table 4 tabulates the optimal policy for all cases, where a dash 


\begin{tabular}{|l|lllll|}
\hline \hline year & 0 & 1 & 2 & 3 & 4 \\
\hline wage $^{1}$ & 6000 & 7000 & 7900 & 8700 & 9400 \\
\hline wage $^{2}$ & 500 & 6000 & 7000 & 7500 & 8000 \\
\hline wage $^{3}$ & 6000 & 7000 & 7400 & 7600 & 7800 \\
\hline \hline
\end{tabular}

Table 3: Alternative compensation schemes

\begin{tabular}{|l|l|l|l|l|l|l|l|l|l|}
\hline \hline training & \multicolumn{3}{|c|}{$I^{0}$} & \multicolumn{3}{c|}{$I^{0.2}$} & \multicolumn{3}{c|}{$I^{0.8}$} \\
\hline attrition & sce 0 & sce 1 & sce 2 & sce 0 & sce 1 & sce 2 & sce 0 & sce 1 & sce 2 \\
\hline base & - & - & - & - & - & - & 0, no & - & 0, no \\
wage $^{1}$ & - & - & - & - & - & - & 1, no & 1, no & 1, no \\
wage $^{2}$ & 0, no & 0, no & 0, no & 0, no & - & 0, no & 0, no & - & 0, no \\
wage $^{3}$ & - & - & - & - & - & - & 1, no & 1, no & 1, no \\
\hline \hline
\end{tabular}

Table 4: Changes in the optimal policy for different wages and training costs

denotes that the optimal policy remained the same as that in the base case, the first number denotes the year in which employees were hired, and no stands for no training. Since in all cases employees are kept until five years old, the table only reports differences in hiring and training policies.

It is clear that the compensation scheme, training costs, as well as attrition patterns can all change the optimal policy. For example as training costs become higher it becomes more desirable to hire one year olds rather than hiring and training zero year olds as seen for the cases of wage $e^{1}$ and $w a g e^{3}$. In some cases the attrition pattern determines whether employees will be trained or not, as seen in the case for wage $e^{2}$ under proportional training costs. Though we do not report dual prices and depreciation for these examples, we can state that for example wage ${ }^{1}$ combined with $I^{0.8}$ ensures that employees do not depreciate throughout their tenure. In contrast, wage $e^{2}$ results in depreciating employees after their first year.

Next, we focus on cyclic demand. Recall that for this type of demand the dual prices were not derived analytically. The examples presented below, will obtain numerical approximations to the value of the infinite horizon problem over a finite horizon. The aim is to illustrate the change in the optimal policy. 


\begin{tabular}{|l|l|l|l|l|l|l|l|l|}
\hline \hline $\begin{array}{l}\text { dec. var. } \\
\text { demand }\end{array}$ & $\begin{array}{l}X^{h}(0) \\
\text { hire }\end{array}$ & $\begin{array}{l}X^{h d}(1) \\
\text { hire and train }\end{array}$ & $\begin{array}{l}X^{k}(1) \\
\text { keep }\end{array}$ & $\begin{array}{l}X^{k}(2) \\
\text { keep }\end{array}$ & $\begin{array}{l}X^{k}(3) \\
\text { keep }\end{array}$ & $\begin{array}{l}X^{k}(4) \\
\text { keep }\end{array}$ & $\begin{array}{l}X^{l}(2) \\
\text { layoff }\end{array}$ & $\begin{array}{l}X^{l}(5) \\
\text { layoff }\end{array}$ \\
\hline 1000 & 65 & 486 & 0 & 10 & 0 & 105 & 65 & 105 \\
1500 & 0 & 0 & 486 & 0 & 10 & 0 & 0 & 0 \\
2000 & 0 & 19 & 0 & 486 & 0 & 10 & 0 & 10 \\
2500 & 86 & 0 & 19 & 0 & 486 & 0 & 0 & 0 \\
3000 & 49 & 10 & 0 & 105 & 0 & 486 & 49 & 486 \\
500 & 0 & 0 & 10 & 0 & 105 & 0 & 0 & 0 \\
\hline \hline
\end{tabular}

Table 5: Optimal Stationary Policy Under Cyclic Demand

Service demand is cyclic and periodic. For simplicity, the trend is assumed to be zero. The example is based on the same parameters as tabulated in Table 1. Demand repeats the following pattern:

$1000,1500,2000,2500,3000,500,1000,1500 \ldots$

Approximating the problem over a 100 period finite horizon, we get the cyclic stationary policy shown in Table 5 for Scenario 0 with no attrition.

Most of the fluctuation in demand is handled by a similarly fluctuating hiring policy. The optimal policy is still the same; to hire employees without any experience, immediately train them and keep them until they retire. Due to the sudden decrease in demand at the end of the cycle, not all employees can be employed with the otherwise optimal policy. We observe that some employees are not trained and among them, some are kept until retirement whereas others are replaced at the end of their second year. In other words, the company chooses not to invest in all of its employees and keeps some of them for shorter periods. This is similar to the common temporary employment practice. Among the workers who are kept until they quit the job, the decision not to train some, is based solely on economic considerations.

We next look at the same example under a attrition pattern as in Scenario 1. Solving over a 100 period finite horizon, we obtain the results shown in Table 6.

Four different replacement cycles are required to cope with cyclical demand. All employees are hired without experience, trained immediately and kept until two, three, four or five year experience levels are reached. This example illustrates an instance where from a purely 


\begin{tabular}{|l|l|l|l|l|l|l|l|l|}
\hline \hline $\begin{array}{l}\text { dec. var. } \\
\text { demand }\end{array}$ & $\begin{array}{l}X^{h d}(1) \\
\text { hire and train }\end{array}$ & $\begin{array}{l}X^{k}(2) \\
\text { keep }\end{array}$ & $\begin{array}{l}X^{k}(3) \\
\text { keep }\end{array}$ & $\begin{array}{l}X^{k}(4) \\
\text { keep }\end{array}$ & $\begin{array}{l}X^{l}(2) \\
\text { layoff }\end{array}$ & $\begin{array}{l}X^{l}(3) \\
\text { layoff }\end{array}$ & $\begin{array}{l}X^{l}(4) \\
\text { layoff }\end{array}$ & $\begin{array}{l}X^{l}(5) \\
\text { layoff }\end{array}$ \\
\hline 1000 & 167 & 0 & 0 & 100 & 0 & 0 & 0 & 95 \\
1500 & 333 & 125 & 0 & 0 & 0 & 0 & 0 & 0 \\
2000 & 174 & 250 & 106 & 0 & 0 & 0 & 0 & 0 \\
2500 & 181 & 130 & 213 & 96 & 0 & 0 & 0 & 91 \\
3000 & 334 & 136 & 111 & 191 & 250 & 4 & 100 & 182 \\
500 & 0 & 0 & 111 & 0 & 0 & 0 & 0 & 0 \\
\hline \hline
\end{tabular}

Table 6: Optimal Stationary Policy Under Cyclic Demand with Attrition

economic standpoint, a seniority based layoff policy frequently used by service firms, is not optimal. Instead policies that resemble early retirement are used. The high attrition rate for zero year olds prevents the use of no training policies that were observed in the above example. By training all its employees the company is avoiding high attrition problems in the first year of employment. This is true as long as the assumption that training changes both productivity and attrition intentions holds.

This Section illustrates several points. First, as the examples in Table 4 show, it is very difficult to make objective policy recommendations without a tool, providing an objective benchmark to compare cases with changes in the raw data. Second, one can see that attrition can change both the optimal replacement cycle and the economic value of human resources to a firm. This underlines the importance of understanding attrition behavior in firms. Given the parallels between attrition and stochastic learning, the same conclusions apply for this case. Finally, whenever one has steady demand, or monotonic growth or contraction for a certain service, human resource value is not impacted by the absolute value of demand. This implies that the valuation only requires an understanding of cost, productivity, and attrition behavior. Firms that operate in environments with business cycles on the other hand need a good qualitative understanding of the demand fluctuations in order to value their human resources. 


\section{Concluding Remarks}

Considering human resources as assets has led to an analysis of investment decisions and appreciation schedules for human capital. The analysis has shown that stationary dual prices can be obtained both for the case with constant or monotonical demand and for the case with cyclical demand. Using the standard economic interpretation of dual prices, we are thus able to characterize the economic value of human resources to the firm.

Interpreting the dual prices as the book value of an employee, one can obtain an appreciation scheme for human resources. Appreciation schedules are managerial accounting tools, which would help to communicate human resource issues within an organization. The inability of human resource departments to quantify the effects of human resource policies on the bottomline of a firm, frequently result in these issues being treated as lower priority. Even for firms who keep track of all the data that would be necessary to develop such appreciation schedules, it is frequently the case that establishing a coherent measure reflecting the impact of human resource policies remains a challenge. The approach herein provides such a measure. The availability of human resource related data is a common difficulty cited in work referring to the human resource accounting literature. While this remains an important challenge, we note that with the adoption of ERP technologies and shared service structures for human resource management, important steps have been taken in the data availability issue.

The discussion in Section 6 illustrates one way of treating uncertainty in learning subsequent to a keep or train decision. For a Markovian case, the analysis illustrates that the impact of stochastic learning on human resource value will be analogous to the impact of attrition in the basic deterministic case. Given the observation that different attrition

patterns can imply changes in human resource value, one can state that whenever it is more appropriate to treat learning as stochastic, understanding the precise nature of this learning is important for human resource valuation. Based on this insight, an important direction for future research can be identified as a detailed modeling and analysis of the stochastic learning case.

In environments where the jobs being considered are unstructured, such that productivity is heavily influenced by personal differences, the assumption of a homogeneous set of employees will become restrictive. Differences in individual performance can be represented by a productivity vector $\mathbf{K}(\theta)$, where $\theta$ is a random variable that models individual differ- 
ences. This modeling approach is similar to the one in [15]. This would constitute a major change in the model, requiring a new approach. Instead, an extension where employees can be in different capability groups as well as different years of experience can be considered. This would imply that $\mathbf{K}$ is a matrix. While heterogeneity is still not at the individual level, this approach could capture major capability differences and could be treated in the mathematical programming framework used in this paper.

An infinite horizon is an abstraction. This problem has been addressed before, in the context of capacity expansion problems (see for example [3]). It would be interesting to study the precise form of demand data in different services, and try to obtain finite horizons over which the problem can be solved equivalently.

\section{Appendix}

Proof of Theorem $1\left\{\alpha^{t-1} \bar{\lambda}\right\}_{t=1}^{\infty}$, and $\left\{\alpha^{t-1} \bar{\mu}\right\}_{t=1}^{\infty}$ are dual feasible since $\bar{\lambda}, \bar{\mu}$ are feasible for $D_{1}(\bar{b}, \bar{\mu}, S)$ and $\left\{X_{t}\right\}_{t=1}^{\infty}$ is primal feasible by definition.

Thus,

$$
\sum_{t=1}^{T} \alpha^{t-1}\left(\mathbf{C X}_{\mathbf{t}}+\alpha \mathbf{D} \mathbf{X}_{\mathbf{t}}\right)+\alpha^{T} \bar{\mu} \mathbf{B} \mathbf{X}_{\mathbf{T}}=\sum_{t=1}^{T} \alpha^{t-1} \bar{\lambda} S_{t}+\bar{\mu} \mathbf{b}_{\mathbf{0}} .
$$

(If $\left\{X_{t}\right\}_{t=1}^{\infty}$ is optimal for the primal problem, then $\left\{X_{t}\right\}_{t=1}^{T}$ is optimal for the T-period approximation) Since $S_{t} \geq 0$ for all $t$, the infinite horizon dual objective converges to a finite value $\left(\lim \sup _{T \rightarrow \infty} \sum_{t=1}^{T} \alpha^{t-1} S_{t}<\infty\right)$.

$$
\lim _{T \rightarrow \infty} \alpha^{T} \bar{\mu} \mathbf{B} \mathbf{X}_{\mathbf{T}}=0
$$

Hence,

$$
\sum_{t=1}^{\infty} \alpha^{t-1}\left(\mathbf{C X}_{\mathbf{t}}+\alpha \mathbf{D} \mathbf{X}_{\mathbf{t}}\right)=\sum_{t=1}^{\infty} \alpha^{t-1} \bar{\lambda} S_{t}+\bar{\mu} \mathbf{b}_{\mathbf{0}}
$$

The infinite-horizon primal objective converges to the same finite value as the dual. 


\section{Proof of Theorem 2 Let}

$$
\begin{aligned}
H & =\left\{i: \bar{\mu}(i)=\left(\tilde{\lambda} K_{i-1}-\left(h_{i-1}+w_{i-1}\right)-\alpha r_{i}\right) \frac{1}{\alpha}, i=1, \ldots, j+1\right\} \\
H D & =\left\{i: \bar{\mu}(i)=\left(\tilde{\lambda} K_{i-1}-\left(h_{i-2}+w_{i-1}+I_{i-2}\right)-\alpha r_{i}\right) \frac{1}{\alpha}, i=2, \ldots, j+1\right\} \\
K & =\left\{i: \bar{\mu}(i)=\left(\tilde{\lambda} K_{i-1}-\left(w_{i-1}-r_{i-1}\right)-\alpha r_{i}+\bar{\mu}(i-1)\right) \frac{1}{\alpha}, i=j+2, \ldots, l-1\right\} \\
D & =\left\{i: \bar{\mu}(i)=\left(\tilde{\lambda} K_{i-1}-\left(w_{i-1}-r_{i-2}+I_{i-2}\right)-\alpha r_{i}+\bar{\mu}(i-2)\right) \frac{1}{\alpha}, i=j+3, \ldots, l-1\right\}
\end{aligned}
$$

Next for $b \in \beta$ define a $4 n-4$ vector $\hat{x}$ by

$$
\hat{x}(i)= \begin{cases}\bar{b}(i-1) & i=k-1, k \in K, k=j+2, \ldots, l \\ 0 & i=k-1, k \notin K, k=j+2, \ldots, l \\ 0 & i=l, \ldots, n-1 \\ \bar{b}(i-2 n) & i=2 n+k-3, k \in D, k=j+3, \ldots, l \\ 0 & i=2 n+k-3, k \notin D, k=j+3, \ldots, l \\ 0 & i=2 n+k-2, k=l, \ldots, n-1 \\ \bar{b}(i-n) & i=n+k, k \in H, k=j \\ 0 & i=n+k, k \neq j, k=0, \ldots, n-1 \\ \bar{b}(i-3 n+2) & i=3 n+k-2, k \in H D, k=j \\ 0 & i=3 n+k-2, k \neq j, k=0, \ldots, n-2\end{cases}
$$

For dual feasibility we must have

$$
\begin{aligned}
\bar{\lambda} \mathbf{K}+\bar{\mu} \mathbf{A} & \leq \mathbf{C}+\alpha \mathbf{D}+\alpha \bar{\mu} \mathbf{B} \\
\bar{\lambda} & \geq 0 \\
\bar{\mu} & \leq 0
\end{aligned}
$$

First note that by definition, $\bar{\lambda}$ will always be positive. We must show that $\bar{\mu}$ as characterized above is nonpositive for all $i$, and that the constraints are satisfied for such $\bar{\mu}$. First rearrange the dual constraint to obtain

$$
\bar{\lambda} \mathbf{K}+\bar{\mu} \mathbf{A}-\mathbf{C}-\alpha \mathbf{D}-\alpha \bar{\mu} \mathbf{B} \leq 0
$$

and observe that for nonpositive $\bar{\mu}$, it is necessary for the left hand side to be nonpositive. Now assume that $\bar{\mu}$ is nonpositive and check if the constraint is satisfied. Setting the left hand sides less than or equal to zero we obtain the following $4 n-4$ conditions.

$$
\begin{aligned}
& \bar{\lambda} \leq\left(-\bar{\mu}(i-1)+w_{i-1}-r_{i-1}+\alpha r_{i}+\alpha \bar{\mu}(i)\right) / K_{i-1}, \quad i=2, \ldots, n \\
& \bar{\lambda} \leq\left(h_{i-1}+w_{i-1}+\alpha r_{i}+\alpha \bar{\mu}(i)\right) / K_{i-1}, \quad i=1, \ldots, n \\
& \bar{\lambda} \leq\left(-\bar{\mu}(i-2)+w_{i-1}+I_{i-2}+r_{i-2}+\alpha r_{i}+\alpha \bar{\mu}(i)\right) / K_{i-2}, \quad i=3, \ldots, n \\
& \bar{\lambda} \leq\left(h_{i-2}+w_{i-1}+I_{i-2}+\alpha r_{i}+\alpha \bar{\mu}(i)\right) / K_{i-1}, \quad i=2, \ldots, n
\end{aligned}
$$


Substituting for $\bar{\mu}(i)$ in the above inequalities we observe that they hold as strict equalities. This implies that the dual constraints are satisfied for $\bar{\mu}$ as characterized before. It remains to show that as characterized, $\bar{\mu}$ cannot be positive. In particular observe that if $\bar{\mu}(i)$ is positive, the characterization of $\bar{\mu}$ implies

$$
\bar{\lambda}>\frac{h_{0}+w_{0}+\ldots+\alpha^{i} r_{i}}{K_{0}+\ldots+\alpha^{i-1} K_{i-1}}
$$

which clearly contradicts the definition of $\bar{\lambda}$. Hence, the constraints for dual feasibility are satisfied.

Having shown that $\bar{\mu}$ is dual feasible, we observe that

$$
(\bar{\lambda} K+\bar{\mu} A-\alpha \bar{\mu} B-C-\alpha D)^{T} \hat{x}=0,
$$

or

$$
\bar{\lambda} K \hat{x}+\bar{\mu} A \hat{x}-\alpha \bar{\mu} B \hat{x}=C \hat{x}+\alpha D \hat{x}
$$

Using the fact that $A \hat{x}=b$ and $K \hat{x}=S$ we get

$$
\bar{\lambda} S+\bar{\mu} b=C \hat{x}+\alpha D \hat{x}+\alpha \bar{\mu} B \hat{x} .
$$

The dual objective is equal to the primal objective. This implies that as defined, $\bar{\lambda}, \bar{\mu}$, and $\hat{x}$ are optimal for the one period dual and primal problems respectively.

Proof of Theorem $3\left\{\alpha^{t-1} \bar{\lambda}_{\tilde{t}}\right\}_{t=0}^{\infty},\left\{\alpha^{t-1} \bar{\mu}_{\tilde{t}}\right\}_{t=0}^{\infty}$ are dual feasible since $\bar{\lambda}, \bar{\mu}$ are feasible for $D_{q}\left(\bar{b}_{j}, \bar{\mu}_{j}, S_{j}\right)$ and $\left\{X_{t}\right\}_{t=1}^{\infty}$ is primal feasible by definition. Thus we get,

$$
\sum_{t=1}^{T} \alpha^{t-1}\left(\mathbf{C X}_{\mathbf{t}}+\alpha \mathbf{D} \mathbf{X}_{\mathbf{t}}\right)+\alpha^{T} \bar{\mu}_{\tilde{T}+1} B X_{T-1}=\sum_{t=1}^{T} \alpha^{t-1} \bar{\lambda}_{\tilde{t}} S_{t}+\overline{\mu_{1}} \mathbf{b}_{\mathbf{0}} .
$$

Based on the prior assumption about the convergence of the $q$ different demand sequences, we observe that the right hand side of the above equation converges to a finite value. Noting that $\lim _{T \rightarrow \infty} \alpha^{T} \bar{\mu}_{\tilde{T}+1} B X_{T-1}=0$, we obtain the equality

$$
\sum_{t=1}^{\infty} \alpha^{t-1}\left(C X_{t}+D X_{t-1}\right)=\sum_{t=1}^{\infty} \alpha^{t-1} \bar{\lambda}_{\tilde{t}} S_{t}+\overline{\mu_{1}} b_{0}
$$

Hence, the infinite horizon primal objective converges to the same finite value as the dual. 


\section{Acknowledgement}

I thank Patrick T. Harker for introducing me to this problem. The article has benefited from comments of three anonymous referees and an associate editor.

\section{References}

[1] Bartholomew, D.J. (1973), Stochastic Models for Social Processes, 2nd ed. New York: Wiley.

[2] Bartholomew,D.J. Forbes, A.F., McClean, S.I. (1991), Statistical Techniques for Manpower Planning, 2nd ed. John Wiley and Sons, New York.

[3] Bean, J.C., and Smith, R.L. (1985), "Optimal capacity expansion over an infinite horizon," Management Science, 31:12 1523- 1532.

[4] Becker, G.S. (1962), "Investment in human capital: a theoretical analysis," The Journal of Political Economy, Supplement: October 9-49.

[5] Bertsekas, D.P. (1987), Dynamic Programming: Deterministic and Stochastic Models, Prentice-Hall, Inc.

[6] Bontis, N., Dragonetti, N.C., Jacobsen, K., and Roos, G. (1999), "The Knowledge Toolbox: A Review of the Tools Available to Measure and Manage Intangible Resources," European Management Journal, 17:4 391-402.

[7] Brummet, R.L., Flamholtz, E., and Pyle, W.C. (1968), "Human resource management: a challenge for accountants," The Accounting Review, American Accounting Association, U.S.A., 217-224.

[8] Conway, R., and Schultz, A. (1959), "The manufacturing process function," Journal of Industrial Engineering, 10:1 39-53.

[9] Dalmahoy, M. (1996), "Putting a value on people", Management Accounting, 74:2 27.

[10] Durso, A. and Donohue, S.F. (1995), "An analytical approach to reshaping the United States Army", Interfaces, 25 109-133. 
[11] Ebert, R.J. (1976), "Aggregate planning with learning curve productivity," Management Science, 23:2 171-182.

[12] The Economist (1993), "The Death of Corporate Loyalty", April 3.

[13] The Economist (1996), "The Year Downsizing Grew Up", December 21.

[14] Evers, J.J.M. (1973), Linear Programming over an Infinite Horizon, Tilburg University Press, Holland.

[15] Fernandez-Gaucherand, E., Jain, S., Lee, H.L., Rao, A.G. and Rao, M.R. (1995), "Improving productivity by periodic perfoemance evaluation: a Bayesian stochastic model", Management Science, 41:10 1669-1678.

[16] Flamholtz, E.G. (1985), Human Resource Accounting: Advances in Concepts, Methods, and Applications, Jossey-Bass, San Francisco.

[17] Fuchs, V.R. (1968) The Service Economy, National Bureau of Economic Research No:87, New York.

[18] Gans, N. and Zhou Y.P. (2002), "Managing learning and turnover in employee staffing", Operations Research, 50:6, 991-1006.

[19] Gass, S.I. (1991), "Military Manpower Planning Models", Computers and Operations Research, 18:1 65-73.

[20] Gerchak, Y. and Golany, B. (2000) "Hiring policies in an uncertain environment: Cost and productivity trade-offs" European Journal of Operational Research, 125 195-204.

[21] Gerchak, Y., Parlar, M., and Sengupta, S.S. (1990), "On manpower planning in the presence of learning," Engineering Costs and Production Economics 20 295-303.

[22] Grinold, R.C. (1971), "Infinite horizon programs," Management Science 18 157-169.

[23] Grinold, R.C. and Hopkins D.S.P. (1973), "Computing optimal solutions for infinitehorizon mathematical programs with a transient stage", Operations Research 21 179187.

[24] Grinold, R.C., and Marshall, K.T. (1977), Manpower Planning Models, Publications in Operations Research Series, Elsevier North-Holland, Inc. 
[25] Hekimian, J.S., and Jones, C.H., (1967), "Put people on your balance sheet," Harvard Business Review, January/February 105-113.

[26] Jaggi, B., and Lau, S. (1974), "Toward a model of human resource valuation," The Accounting Review, April 321-329.

[27] Jones, P.C., Zydiak, J.L., and Hopp, W.J. (1988), "Stationary dual prices and depreciation," Mathematical Programming 41 357- 366.

[28] Jones, P.C., Zydiak, J.L., and Hopp, W.J. (1992) "Capital Asset Valuation and Depreciation for Stochastically Deteriorating Equipment," The Engineering Economist, 38:1 19-30.

[29] Katz, R. (1969), "Understanding and applying learning curves," Automation, 16:11 $50-53$.

[30] Khoshnevis, B. and Wolfe, P.M. (1983), "An aggregate production planning model incorporating dynamic productivity: part I. Model development," IIE Transactions, 15 111-118.

[31] Koretz (1999), "Quick to Fire Quick to Hire", Business Week, May 31: 3631.

[32] Kostiuk, P.F., and Follmann, D.A. (1989) "Learning curves, personal characteristics, and job performance," Journal of Labor Economics, 7:2 129-146.

[33] Lau, A.H., and Lau, H. (1978), "Some proposed approaches for writing off capitalized human resource assets," Journal of Accounting Research, Spring 80-102.

[34] Lesser and Prusak (2001), "Preserving Knowledge in an Uncertain World", MIT Sloan Management Review, Fall 101-102.

[35] Lev, B., and Schwartz, S. (1971), "On the use of the economic concept of human capital in financial statements," The Accounting Review, January 103-113.

[36] Liang, T.T. and Buclatin, B.B. (1988), "Improving the utilization of training resources through optimal personnel assignment in the U.S. Navy", European Journal of Operational Research, 33 183-190.

[37] Longenecker, C.O. and Ariss, S.S. (2004), "Who goes and who stays?", Industrial Management, 46:3 8-13. 
[38] Lovejoy, W.S. (2000), Integrated Operations, text in preparation, University of Michigan, Ann Arbor, Michigan.

[39] Mazzola, J.B., McCardle, K.F. (1997), "The stochastic learning curve: optimal production in the presence of learning-curve uncertainty", Operations Research, 45:3 440-450.

[40] McLaughlin, K.J. (1990), "General productivity growth in a theory of quits and layoffs," Journal of Labor Economics, 8:1 75-98.

[41] Nusbaumer, J. (1987) Services in the Global Market Kluwer Academic Publishers, Boston.

[42] Orrbeck, M.G., Schuette, D.R., and Thompson, H.E. (1968), "The effect of worker productivity on production smoothing," Management Science, 14 B332-B342.

[43] Palmer, C. (2003) "The math of workforce reductions", The Daily Reporter Milwaukee, June 23, p1.

[44] Pauker, A.M. and Gallagher, D.J. (1999), "Valuing assets that count: Integrity, Innovation, Individuals" The Secured Lender, 55:1 38-42.

[45] Phillips, J.D. (1990), "The price on turnover," Personnel Journal, December 58-61.

[46] Quigley, M.O., and Henshaw, T.J. (1987), "A model to simulate the effects of work force dynamics on compensation policy," Strategic Human Resource Planning Applications, R.J. Niehaus ed., Plenum Press, 185-194.

[47] Rogow, R.B., and Edmonds, C.P. (1988), "Tallying employees as assets," Personnel Administrator, June 168-170.

[48] Sackmann, S.A., Flamholtz, E.G., and Bullen, M.L. (1989), "Human resource accounting: a state-of-the-art review," Journal of Accounting Literature, 8 235-264.

[49] Scarpello, V., and Theeke, H.A. (1989), "Human resource accounting: a measured critique," Journal of Accounting Literature, 8 265-280.

[50] Segalla, M., Jacobs-Belschak, G., Muller, C. (2001) "Cultural influences on employee termination decisions: Firing the good, average or the old?", European Management Journal, 19:1 58-72.

[51] Shroad, V.J. (1964), "Control of labor costs through the use of learning curves," N.A.A. Bulletin, 46:2 15-20. 
[52] Steffy, B.D., and Maurer, S.D. (1988), "Conceptualizing and measuring the economic effectiveness of human resource activities," Academy of Management Review, 13:2 271286 .

[53] Sveiby, K.E. (1997), The New Organizational Wealth: Managing and Measuring Knowledge-Based Assets, Berrett-Koehler Publishers, Inc.

[54] Ugwuowo, F.I., McClean, S.I. (2000), "Modelling heterogeneity in a manpower system: a review," Applied Stochastic Models in Business and Industry, 16 99-110.

[55] Vajda (1978) Mathematics of Manpower Planning, Chicester, England, New York.

[56] Wertmann, L. (1959), "Putting learning curves to work," Tool Engineer, 43:2 99-102.

[57] Yelle, L.E. (1979), "The learning curve: historical review and comprehensive survey," Decision Sciences, 10 302-328. 